\title{
Path-Independent integral for fracture of solids under combined electrochemical and mechanical loadings
}

\author{
qu, jianmin, j-qu@northwestern.edu, northwestern university
}

\begin{abstract}
It is well known that invariant or path-independent integrals provide a powerful tool in the study of crack initiation and growth in functional materials. It is shown in this article that the classical J-integral is no longer path-independent when the solid is under combined electrochemical and mechanical loading. A modified J-integral is derived that is path-independent under electro-chemo-mechanical equilibrium. To illustrate the utilization of this modified J-integral, the delamination of a thin elastic film from an infinitely thick elastic substrate is considered under chemo-mechanical equilibrium. Within the membrane approximation, the problem reduces to finding stress and solute concentration in a thin membrane adhered to the substrate. By adopting a linearized chemo-mechanical model, this problem is solved semi-analytically. The solution is then used to demonstrate that the classical J-integral is path-dependent, whereas the modified J-integral derived in this article is path-independent.
\end{abstract}

